\title{
Supporting Information for - Assessing Economic Modulation of Future Critical Materials Use: The Case of Automotive-Related Platinum Group Metals
}

Jingshu Zhang1, Mark P. Everson ${ }^{2}$, Timothy J. Wallington ${ }^{2}$, Frank R. Field III' ${ }^{1}$, Richard Roth ${ }^{1}$, Randolph E. Kirchain ${ }^{*}$

${ }^{1}$ Materials Systems Laboratory, Massachusetts Institute of Technology, Cambridge, MA, USA

${ }^{2}$ Ford Motor Company's Research and Innovation Center in Dearborn, MI, USA

* Materials Systems Laboratory, Massachusetts Institute of Technology

77 Massachusetts Ave, E38 - 432

Cambridge, MA 02139

(617) 253-4258

kirchain@mit.edu

\section{Contents}

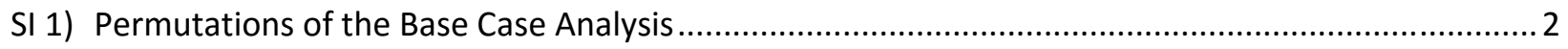

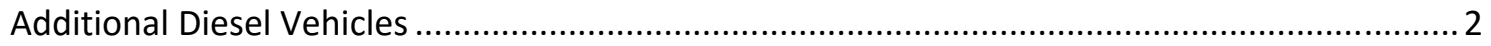

Additional Alternative Propulsion Systems ........................................................................ 2

SI 2) Parameters and Fit Statistics for Substitution Models ............................................................

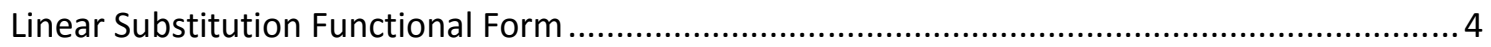

Generalized Inverse Substitution Functional Form ................................................................ 4

SI 3) PGM Loading After Adjusting for Learning and Volume: TWC ..................................................... 5

SI 4) TWC Weight Change and Learning Rate for Different Standard Transitions................................... 7

SI 5) PGM Loading after Adjusting for Learning and Volume: DOC ................................................ 8

SI 6) DOC Weight Change and Learning Rate for Different Standard Transitions ................................... 10

SI 7) Annual Assumed Vehicle Sales by Type (values in millions) ......................................................... 11

SI 8) Annual Assumed Vehicle Sales by Region (values in millions) ....................................................... 12

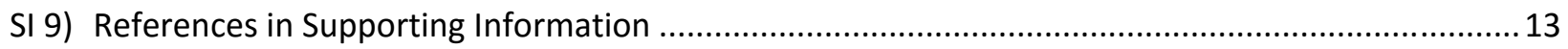




\section{SI 1) Permutations of the Base Case Analysis}

\section{Additional Diesel Vehicles}

Diesel vehicles are more fuel efficient than gasoline vehicles and it is possible that their market share will increase. We consider a High Diesel Case where the absolute volume of diesel light duty vehicle sales (11.7 million in 2013) is assumed to double by 2030 at the expense of gasoline vehicle sales.

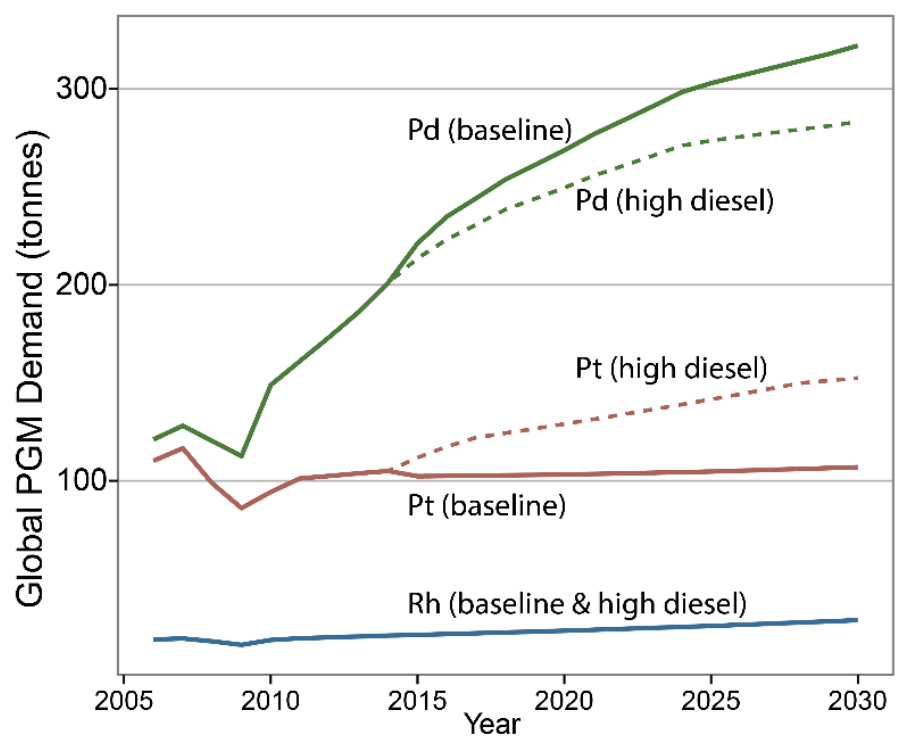

Figure SI.1: Modeled global automotive Pd, Pt, and Rh demand for the Baseline Case (solid lines) and for the High Diesel Case (dashed lines).

The results from the High Diesel Case are compared to those in the Base Case in Figure SI.1. Diesel exhaust control systems use more Pt than Pd, whereas those for gasoline engines use more Pd than Pt, therefore an increase of diesel vehicles on the road at the expense of gasoline vehicles sales will increase Pt demand at the expense of Pd demand. In the High Diesel Case the global demand of Pd stagnates by about 2025 while the global demand of Pt increases throughout the period up to 2030. Compared to the Base Case, in 2030 the Pt demand increases (by about 45 tonnes, or $\sim 42 \%$ ) and the Pd demand decreases decreases by about 39 tonnes (or $12 \%$ ).

\section{Additional Alternative Propulsion Systems}

In addition, we consider two Alternative Technology Vehicle Cases, where first the battery electric vehicle (BEV) and then the fuel cell vehicle (FCV) market shares are assumed to increase by 2030 at the expense of gasoline vehicles.

Figure SI.2 shows the modeled PGM global demand calculated for a case where the sales of electric vehicles increase 10-fold by 2030; from 200,000 in 2013 to 2.0 million in 2030. 


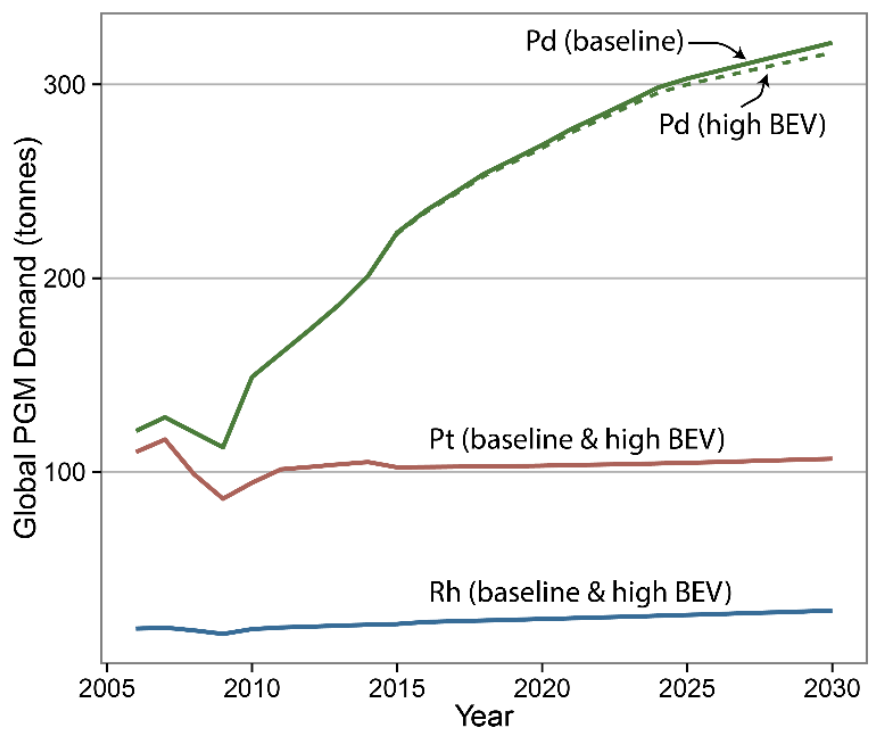

Figure SI.2: Modeled global Pd, Pt, and Rh demand the Baseline Case (solid lines) and for the High BEV Case (dashed lines).

From Figure SI.2 it is clear that this rate of increase of BEV sales (sales increase of 15\%/year) would not lead to a major impact on the global automotive-related PGM demand. The model suggests that the global $\mathrm{Pt}, \mathrm{Pd}$, and $\mathrm{Rh}$ demand would decrease by about $0.25 \%, 1.5 \%$, and $1.4 \%$, respectively.

In the final scenario, we assume the market share of fuel cell vehicles will increase from the current essentially zero volume to 110,000 units $(0.1 \%$ market share) by 2030 at the expense of gasoline vehicles. Fuel cell vehicles do not use catalytic converters but they do use Pt catalyst in the fuel cell stack. The Department of Energy target for Pt use in proton exchange membrane fuel cells is $0.125 \mathrm{~g} / \mathrm{kW}$ (see D. Papageorgopoulos, "DOE Hydrogen and Fuel Cells Program: FY 2014 Annual Progress Report " available at http://hydrogen.energy.gov/pdfs/progress14/v 0 papageorgopoulos 2014.pdf accessed March 15, 2015.) which for a typical $80 \mathrm{~kW}$ light duty vehicle equates to the use of $10 \mathrm{~g}$ Pt per vehicle which we assume will be achieved by 2030.

The results of this simulation indicate that a modest market share of fuel cell vehicles in 2030 would have only a minor impact on the global automotive-related PGM demand for Pd $(0.1 \%$ decrease compared to Base Case) and Rh (0.1\% decrease), and a slightly greater impact on the global demand for Pt $(0.6 \%$ increase). No plot of these results is provided because this scale of difference is not resolvable on a reasonable scale figure. 


\section{SI 2) Parameters and Fit Statistics for Substitution Models}

\section{Linear Substitution Functional Form}

\begin{tabular}{l|cc|cc}
\multicolumn{4}{c}{$M^{P t}=\alpha\left(M^{P d}\right)+\beta$} \\
\cline { 3 - 3 } & Value & $\mathrm{p}$ & Value & $\mathrm{p}$ \\
\hline \hline$\alpha$ & -0.93 & $<0.0001$ & -1.21 & 0.00016 \\
$\beta$ & 3.29 & $<0.0001$ & 3.50 & $<0.0001$ \\
\hline $\mathrm{R}^{2}$ & & & & \\
SER & 0.75 & & 0.51 & \\
\hline df & 0.66 & & 0.43 & \\
& 22 & & 19 &
\end{tabular}

Table SI.1. Description of the linear substitution functions used in this work. $\alpha$ and $\beta$ represent the parameters used for the equations describing Pt to Pd substitution for the TWC and DOC. Key statistics about the model performance are provided including the coefficient of determination $\left(R^{2}\right)$ and standard error of the regression (SER). df is the degrees of freedom of the regression model.

\section{Generalized Inverse Substitution Functional Form}

$$
M^{P t}=\frac{h}{k+\left(M^{P d}+M^{R h}\right)}+l
$$

\begin{tabular}{l|rr|cr} 
& \multicolumn{2}{|c|}{ TWC } & \multicolumn{2}{c}{ DOC } \\
& Value & $\mathrm{p}$ & Value & $\mathrm{p}$ \\
\hline \hline$h$ & 17.03 & 0.042 & 7.34 & 0.0002 \\
$k$ & 2.22 & 0.013 & 2.08 & 0.001 \\
$l$ & -2.77 & 0.009 & -- & -- \\
SER & & & & \\
df & 0.40 & & 0.44 &
\end{tabular}

Table SI. 2. Description of the generalized inverse substitution functions used in this work. $h$, $k$, and $l$ represent the parameters used for the equations describing Pt to Pd and Rh substitution for the TWC and DOC. Key statistics about the model performance are provided including the coefficient of determination $\left(R^{2}\right)$ and standard error of the regression (SER). df is the degrees of freedom of the regression model. Note: None of the DOCs included in the dataset for this paper contained rhodium. As such, the term $M^{R h}$ in the generalized form was omitted $\left(M^{R h}=0\right)$ for the DOC. 


\section{SI 3) PGM Loading After Adjusting for Learning and Volume: TWC}

Table SI.3. PGM Loading after adjusting for learning and volume for the TWC

\begin{tabular}{|c|c|c|c|c|c|c|c|c|c|c|c|c|c|c|}
\hline Source & Standard & $\begin{array}{c}\mathrm{CV}^{+} \\
(i) \\
\end{array}$ & $\begin{array}{c}\mathrm{ED}^{\ddagger} \\
(I) \\
\end{array}$ & $\begin{array}{c}\mathrm{Pt} \\
(\mathrm{g} / I) \\
\end{array}$ & $\begin{array}{c}\mathrm{Pd} \\
(\mathrm{g} / /)\end{array}$ & $\begin{array}{c}\mathrm{Rh} \\
(\mathrm{g} / \mathrm{I}) \\
\end{array}$ & $\begin{array}{l}\mathrm{Pt}(\mathrm{g}) \\
M_{t p}^{P t^{\wedge}} \\
\end{array}$ & $\begin{array}{c}\mathrm{Pd}(\mathrm{g}) \\
M_{t p}^{P d} \\
\end{array}$ & $\begin{array}{l}\mathrm{Rh}(\mathrm{g}) \\
M_{t p}^{R h^{\wedge}} \\
\end{array}$ & $\begin{array}{c}\operatorname{PGM}(\mathrm{g}) \\
M_{t p}^{t o t^{\wedge}}\end{array}$ & $\begin{array}{c}\mathrm{Pt}(\mathrm{g}) \\
M_{\text {iso }}^{P t}{ }^{\wedge} \\
\end{array}$ & $\begin{array}{l}\mathrm{Pd}(\mathrm{g}) \\
M_{\text {iso }}^{P d}{ }^{\wedge} \\
\end{array}$ & $\begin{array}{l}\operatorname{Rh}(\mathrm{g}) \\
M_{i s{ }^{R \wedge}}^{R h} \\
\end{array}$ & $\begin{array}{c}\text { PGM (g) } \\
M_{i s o}^{\text {tot }} \\
\end{array}$ \\
\hline $\begin{array}{l}\text { ICCT } \\
2012 *[1]\end{array}$ & Euro 1 & 1.60 & 2.00 & 0.10 & 0.50 & 0.10 & 0.16 & 0.80 & 0.16 & 1.12 & 0.55 & 2.76 & 0.55 & 3.86 \\
\hline $\begin{array}{l}\text { Zammit } \\
2011 \text { [2] }\end{array}$ & Euro 1 & 0.65 & 1.00 & 0.00 & 2.20 & 0.11 & 0.00 & 1.43 & 0.07 & 1.50 & 0.00 & 3.95 & 0.20 & 4.14 \\
\hline $\begin{array}{l}\text { ICCT } \\
2012 *[1]\end{array}$ & Euro 2 & 1.70 & 2.00 & 0.10 & 0.50 & 0.10 & 0.17 & 0.85 & 0.17 & 1.19 & 0.44 & 2.19 & 0.44 & 3.07 \\
\hline $\begin{array}{l}\text { Theis } \\
2012 *[3]\end{array}$ & Euro 2 & 1.50 & 2.00 & 1.18 & 0.23 & 0.00 & 1.77 & 0.35 & 0.00 & 2.12 & 3.88 & 0.76 & 0.00 & 4.63 \\
\hline $\begin{array}{l}\text { ICCT } \\
2012 *[1]\end{array}$ & Euro 3 & 1.80 & 2.00 & 0.10 & 0.60 & 0.10 & 0.18 & 1.08 & 0.18 & 1.44 & 0.35 & 2.09 & 0.35 & 2.78 \\
\hline $\begin{array}{l}\text { Theis } \\
2012 *[3]\end{array}$ & Euro 3 & 1.50 & 2.00 & 0.00 & 2.12 & 0.00 & 0.00 & 3.18 & 0.00 & 3.18 & 0.00 & 4.25 & 0.00 & 4.25 \\
\hline $\begin{array}{l}\text { Chatterjee } \\
2001^{* *}[4]\end{array}$ & Euro 3 & 2.00 & 2.00 & 0.00 & 1.54 & 0.31 & 0.00 & 3.08 & 0.62 & 3.70 & 0.00 & 3.41 & 0.69 & 4.10 \\
\hline $\begin{array}{l}\text { Collins } \\
2002 \text { [5] }\end{array}$ & Euro 3 & 1.90 & 2.00 & 0.00 & 1.11 & 0.19 & 0.00 & 2.11 & 0.36 & 2.47 & 0.00 & 3.67 & 0.63 & 4.30 \\
\hline $\begin{array}{l}\text { ICCT } \\
2012 *[1]\end{array}$ & Euro 4 & 1.90 & 2.00 & 0.10 & 0.60 & 0.10 & 0.19 & 1.14 & 0.19 & 1.52 & 0.38 & 2.27 & 0.38 & 3.02 \\
\hline $\begin{array}{l}\text { Mitchell } \\
2005 \text { [6] }\end{array}$ & Euro 4 & 1.30 & 1.50 & 0.00 & 0.81 & 0.15 & 0.00 & 1.05 & 0.19 & 1.24 & 0.00 & 2.39 & 0.43 & 2.82 \\
\hline $\begin{array}{l}\text { Zammit } \\
2011 \text { [2] }\end{array}$ & Euro 4 & 0.65 & 1.00 & 0.00 & 2.95 & 0.14 & 0.00 & 1.92 & 0.09 & 2.01 & 0.00 & 2.80 & 0.13 & 2.93 \\
\hline $\begin{array}{l}\text { Theis } \\
2012 *[3]\end{array}$ & Euro 4 & 1.50 & 2.00 & 0.14 & 1.84 & 0.14 & 0.21 & 2.76 & 0.21 & 3.18 & 0.31 & 4.03 & 0.31 & 4.64 \\
\hline $\begin{array}{l}\text { Matsuzono } \\
2012 \text { [7] }\end{array}$ & Euro 4 & 2.40 & 2.50 & 0.00 & 1.85 & 0.00 & 0.00 & 4.44 & 0.00 & 4.44 & 0.00 & 3.86 & 0.00 & 3.86 \\
\hline $\begin{array}{l}\text { Hangas } \\
2006 \text { [8] }\end{array}$ & Euro 4 & 2.00 & 2.00 & 1.23 & 0.00 & 0.25 & 2.46 & 0.00 & 0.50 & 2.96 & 3.59 & 0.00 & 0.73 & 4.32 \\
\hline
\end{tabular}




\begin{tabular}{|c|c|c|c|c|c|c|c|c|c|c|c|c|c|c|}
\hline Source & Standard & $\begin{array}{c}\mathrm{CV}^{+} \\
(i) \\
\end{array}$ & $\begin{array}{c}\mathrm{ED}^{\ddagger} \\
(I)\end{array}$ & $\begin{array}{c}\mathrm{Pt} \\
(\mathrm{g} / \mathrm{l})\end{array}$ & $\begin{array}{c}\mathrm{Pd} \\
(\mathrm{g} / \mathrm{I}) \\
\end{array}$ & $\begin{array}{c}\mathrm{Rh} \\
(\mathrm{g} / /)\end{array}$ & $\begin{array}{l}\mathrm{Pt}(\mathrm{g}) \\
M_{t p}^{P t^{\wedge}} \\
\end{array}$ & $\begin{array}{c}\mathrm{Pd}(\mathrm{g}) \\
M_{t p}^{P d} \\
\end{array}$ & $\begin{array}{l}\mathrm{Rh}(\mathrm{g}) \\
M_{t p}^{R h^{\wedge}} \\
\end{array}$ & $\begin{array}{c}\operatorname{PGM}(\mathrm{g}) \\
M_{t p}^{t o t^{\wedge}}\end{array}$ & $\begin{array}{l}\text { Pt (g) } \\
M_{\text {iso }}^{P t}{ }^{\wedge} \\
\end{array}$ & $\begin{array}{l}\operatorname{Pd}(\mathrm{g}) \\
M_{\text {iso }}^{P d}{ }^{\wedge} \\
\end{array}$ & $\begin{array}{l}\text { Rh (g) } \\
M_{i s S^{\wedge \wedge}}^{R h^{\prime}} \\
\end{array}$ & $\begin{array}{c}\text { PGM (g) } \\
M_{\text {iso }}^{\text {tot }} \text { (^ } \\
\end{array}$ \\
\hline $\begin{array}{l}\text { Collins } \\
2002 \text { [5] }\end{array}$ & Euro 4 & 2.40 & 2.50 & 0.00 & 1.11 & 0.19 & 0.00 & 2.66 & 0.46 & 3.12 & 0.00 & 3.89 & 0.67 & 4.55 \\
\hline $\begin{array}{l}\text { ICCT } \\
2012 *[1]\end{array}$ & Euro 5 & 2.00 & 2.00 & 0.10 & 0.70 & 0.10 & 0.20 & 1.40 & 0.20 & 1.80 & 0.32 & 2.27 & 0.32 & 2.92 \\
\hline $\begin{array}{l}\text { Mitchell } \\
2005 \text { [6] }\end{array}$ & Euro 5 & 1.30 & 2.00 & 0.00 & 2.50 & 0.36 & 0.00 & 3.25 & 0.46 & 3.71 & 0.00 & 3.66 & 0.52 & 4.18 \\
\hline $\begin{array}{l}\text { Zammit } \\
2012 \text { [2] }\end{array}$ & Euro 5 & 2.00 & 2.00 & 0.00 & 1.30 & 0.00 & 0.00 & 2.60 & 0.00 & 2.60 & 0.00 & 3.85 & 0.00 & 3.85 \\
\hline $\begin{array}{l}\text { Theis } \\
2012 * \text { [3] }\end{array}$ & Euro 5 & 1.50 & 2.00 & 0.35 & 1.41 & 0.35 & 0.53 & 2.12 & 0.53 & 3.17 & 0.59 & 2.38 & 0.59 & 3.57 \\
\hline $\begin{array}{l}\text { ICCT } \\
2012 *[1]\end{array}$ & Euro 6 & 2.00 & 2.00 & 0.10 & 0.70 & 0.10 & 0.20 & 1.40 & 0.20 & 1.80 & 0.44 & 3.05 & 0.44 & 3.92 \\
\hline $\begin{array}{l}\text { Zammit } \\
2011 \text { [2] }\end{array}$ & Euro 6 & 0.65 & 1.00 & 0.00 & 7.00 & 0.00 & 0.00 & 4.55 & 0.00 & 4.55 & 0.00 & 3.96 & 0.00 & 3.96 \\
\hline $\begin{array}{l}\text { Zammit } \\
2012 \text { [2] }\end{array}$ & Euro 6 & 2.00 & 2.00 & 0.00 & 2.10 & 0.00 & 0.00 & 4.20 & 0.00 & 4.20 & 0.00 & 3.65 & 0.00 & 3.65 \\
\hline $\begin{array}{l}\text { Theis } \\
2012 * \text { [3] }\end{array}$ & Euro 6 & 1.50 & 2.00 & 3.37 & 0.00 & 0.00 & 5.06 & 0.00 & 0.00 & 5.06 & 4.40 & 0.00 & 0.00 & 4.40 \\
\hline $\begin{array}{l}\text { Matsuzono } \\
2012 \text { [7] }\end{array}$ & Euro 6 & 2.40 & 2.50 & 0.00 & 1.60 & 0.40 & 0.00 & 3.84 & 0.96 & 4.80 & 0.00 & 3.34 & 0.84 & 4.18 \\
\hline
\end{tabular}

*: loadings specifically designated to a particular standard, see text

**: no loading comparison is available

The regulation standards for the rest of the data are inferred.

$+\mathrm{CV}=$ catalyst volume (in litres)

$\ddagger E D$ = engine displacement (in litres)

$\wedge M_{t p}^{x}=$ Mass of $\mathrm{x}$ reported in literature

$\wedge \wedge M_{i s o}^{x}=$ Equivalent iso-performance mass of $\mathrm{x}$, adjusted to account for technological progress. 


\section{SI 4) TWC Weight Change and Learning Rate for Different Standard Transitions}

Table SI.4. TWC Weight Change and Learning Rate for Different Standard Transitions

\begin{tabular}{lcccc}
\hline \multicolumn{1}{c}{ Standard Transition } & $\begin{array}{c}\text { Delta Weight } \\
(\mathrm{g})\end{array}$ & $\begin{array}{c}\text { Learning Rate } \\
\%\end{array}$ & $\begin{array}{c}\text { Delta Weight } \\
(\mathrm{g})^{*}\end{array}$ & $\begin{array}{c}\text { Learning Rate } \\
\%^{*}\end{array}$ \\
\hline Euro1-Euro2 & 0.14 & 3.3 & 0.07 & 1.7 \\
\hline Euro2-Euro3 & 0.50 & 8.4 & 0.25 & 4.6 \\
\hline Euro3-Euro4 & 0.16 & 3.0 & 0.08 & 1.2 \\
\hline Euro4-Euro5 & 0.18 & 3.4 & 0.28 & 5.1 \\
Euro5-Euro6 & 0.33 & 7.3 & 0.30 & 6.8 \\
Average & 0.26 & 5.1 & 0.25 & 5.0 \\
\hline
\end{tabular}

*: values calculated with papers that explicitly designate a particular standard for a specific loading. 
SI 5) PGM Loading after Adjusting for Learning and Volume: DOC

Table SI.5. PGM Loading after Adjusting for Learning and Volume for the DOC

\begin{tabular}{|c|c|c|c|c|c|c|c|c|c|c|c|c|c|c|}
\hline Source & Standard & $\begin{array}{c}\mathrm{CV}^{+} \\
(i) \\
\end{array}$ & $\begin{array}{c}\mathrm{ED}^{\ddagger} \\
(I) \\
\end{array}$ & $\begin{array}{c}\mathrm{Pt} \\
(\mathrm{g} / /) \\
\end{array}$ & $\begin{array}{c}\mathrm{Pd} \\
(\mathrm{g} / /)\end{array}$ & $\begin{array}{c}\mathrm{Rh} \\
(\mathrm{g} / \mathrm{l})\end{array}$ & $\begin{array}{l}\mathrm{Pt}(\mathrm{g}) \\
M_{t p}^{P t^{\wedge}} \\
\end{array}$ & $\begin{array}{c}\mathrm{Pd}(\mathrm{g}) \\
M_{t p}^{P d_{\wedge}} \\
\end{array}$ & $\begin{array}{l}\mathrm{Rh}(\mathrm{g}) \\
M_{t p}^{R h^{\wedge}} \\
\end{array}$ & $\begin{array}{c}\operatorname{PGM}(\mathrm{g}) \\
M_{t p}^{t o t^{\wedge}} \\
\end{array}$ & $\begin{array}{c}\mathrm{Pt}(\mathrm{g}) \\
M_{i s o}^{P t} \wedge \\
\end{array}$ & $\begin{array}{l}\mathrm{Pd}(\mathrm{g}) \\
M_{\text {iso }}^{P d}{ }^{\wedge \wedge} \\
\end{array}$ & $\begin{array}{l}\mathrm{Rh}(\mathrm{g}) \\
M_{i s S^{\wedge \wedge}}^{R h^{\wedge}} \\
\end{array}$ & $\begin{array}{c}\text { PGM (g) } \\
M_{\text {iso }}^{\text {ton }} \\
\end{array}$ \\
\hline $\begin{array}{l}\text { Glover } \\
\text { 2011[9] }\end{array}$ & Euro 3 & 1.20 & 2.00 & 1.81 & 0 & 0 & 2.17 & 0 & 0 & 2.17 & 3.26 & 0.00 & 0.00 & 3.26 \\
\hline $\begin{array}{l}\text { Han } \\
2007 \text { [10] }\end{array}$ & Euro 3 & 0.60 & 1.50 & 4 & 0 & 0 & 2.4 & 0 & 0 & 2.4 & 3.15 & 0.00 & 0.00 & 3.15 \\
\hline $\begin{array}{l}\text { Kallinen } \\
2009 *[11]\end{array}$ & Euro 3 & 2.40 & 3.00 & 0.89 & 0.22 & 0 & 2.14 & 0.53 & 0 & 2.66 & 2.80 & 0.69 & 0.00 & 3.49 \\
\hline $\begin{array}{l}\text { ICCT } \\
2012 \text { [1] }\end{array}$ & Euro 4 & 1.80 & 2.00 & 0.66 & 0.33 & 0 & 1.19 & 0.59 & 0 & 1.78 & 1.78 & 0.89 & 0.00 & 2.67 \\
\hline $\begin{array}{l}\text { Glover } \\
\text { 2011[9] }\end{array}$ & Euro 4 & 1.20 & 2.00 & 1.59 & 0.23 & 0 & 1.91 & 0.28 & 0 & 2.18 & 2.86 & 0.41 & 0.00 & 3.28 \\
\hline $\begin{array}{l}\text { Han } \\
\text { 2007[10] }\end{array}$ & Euro 4 & 0.60 & 1.50 & 3 & 1 & 0 & 1.80 & 0.6 & 0 & 2.4 & 2.70 & 0.90 & 0.00 & 3.60 \\
\hline $\begin{array}{l}\text { Kim } \\
2011 *[12]\end{array}$ & Euro 4 & 1.20 & 2.00 & 1.85 & 0 & 0 & 2.22 & 0 & 0 & 2.22 & 3.33 & 0.00 & 0.00 & 3.33 \\
\hline $\begin{array}{l}\text { Desai } \\
2010[13]\end{array}$ & Euro 4 & 1.60 & 2.50 & 1.19 & 0.3 & 0 & 1.90 & 0.48 & 0 & 2.38 & 2.86 & 0.72 & 0.00 & 3.58 \\
\hline $\begin{array}{l}\text { Kallinen } \\
2009 *[11]\end{array}$ & Euro 4 & 2.40 & 3.00 & 1.48 & 0 & 0 & 3.55 & 0 & 0 & 3.55 & 3.80 & 0.00 & 0.00 & 3.80 \\
\hline $\begin{array}{l}\text { Wiebenga } \\
2012^{* *}[14]\end{array}$ & Euro 5 & 1.80 & 2.5 & 1.59 & 0.23 & 0 & 2.86 & 0.41 & 0 & 3.28 & 3.58 & 0.52 & 0.00 & 4.10 \\
\hline $\begin{array}{l}\text { Glover } \\
\text { 2011[9] }\end{array}$ & Euro 5 & 0.80 & 2.00 & 3.35 & 0.84 & 0 & 2.68 & 0.67 & 0 & 3.35 & 3.35 & 0.84 & 0.00 & 4.19 \\
\hline $\begin{array}{l}\text { Han } \\
\text { 2007[10] }\end{array}$ & Euro 5 & 0.60 & 1.50 & 3.2 & 1.1 & 0 & 1.92 & 0.66 & 0 & 2.58 & 2.05 & 0.71 & 0.00 & 2.76 \\
\hline $\begin{array}{l}\text { Kim } \\
2011[12]\end{array}$ & Euro 5 & 1.60 & 2.00 & 1.24 & 0.61 & 0 & 1.98 & 0.98 & 0 & 2.96 & 2.27 & 1.12 & 0.00 & 3.39 \\
\hline $\begin{array}{l}\text { Johnson } \\
2010[15]\end{array}$ & Euro 5 & 0.80 & 1.50 & 2.1 & 1.05 & 0 & 1.68 & 0.84 & 0 & 2.52 & 1.80 & 0.90 & 0.00 & 2.70 \\
\hline
\end{tabular}




\begin{tabular}{|c|c|c|c|c|c|c|c|c|c|c|c|c|c|c|}
\hline Source & Standard & $\begin{array}{c}\mathrm{CV}^{+} \\
(i)\end{array}$ & $\begin{array}{c}\mathrm{ED}^{\ddagger} \\
(I)\end{array}$ & $\begin{array}{c}\mathrm{Pt} \\
(\mathrm{g} / \mathrm{I})\end{array}$ & $\begin{array}{c}\mathrm{Pd} \\
(\mathrm{g} / \mathrm{l})\end{array}$ & $\begin{array}{c}\mathrm{Rh} \\
(\mathrm{g} / \mathrm{l})\end{array}$ & $\begin{array}{l}\mathrm{Pt}(\mathrm{g}) \\
M_{t p}^{P t^{\wedge}}\end{array}$ & $\begin{array}{l}\mathrm{Pd}(\mathrm{g}) \\
M_{t p}^{P d_{\wedge}}\end{array}$ & $\begin{array}{l}\mathrm{Rh}(\mathrm{g}) \\
M_{t p}^{R h^{\wedge}}\end{array}$ & $\begin{array}{c}\text { PGM(g) } \\
M_{t p}^{t o t^{\wedge}}\end{array}$ & $\begin{array}{c}\mathrm{Pt}(\mathrm{g}) \\
M_{\text {iso }}^{P t} \wedge \\
\end{array}$ & $\begin{array}{l}\mathrm{Pd}(\mathrm{g}) \\
M_{i s o}^{P d \wedge}\end{array}$ & $\begin{array}{l}\operatorname{Rh}(\mathrm{g}) \\
M_{i s o}^{R h^{\wedge}}\end{array}$ & $\begin{array}{c}\text { PGM (g) } \\
M_{\text {ist }}^{\text {tot }} \text { (^ }\end{array}$ \\
\hline $\begin{array}{l}\text { Desai } \\
2010[13]\end{array}$ & Euro 5 & 2.00 & 2.50 & 1.19 & 0.3 & 0 & 2.38 & 0.60 & 0 & 2.98 & 2.55 & 0.64 & 0.00 & 3.19 \\
\hline $\begin{array}{l}\text { Kallinen } \\
2009^{*}[11]\end{array}$ & Euro 5 & 1.20 & 2.50 & 2.07 & 0.52 & 0 & 2.48 & 0.62 & 0 & 3.11 & 2.66 & 0.66 & 0.00 & 3.32 \\
\hline $\begin{array}{l}\text { Glover } \\
\text { 2011[9] }\end{array}$ & Euro 6 & 0.80 & 2.00 & 4.19 & 0 & 0 & 3.35 & 0 & 0 & 3.35 & 3.35 & 0.00 & 0.00 & 3.35 \\
\hline $\begin{array}{l}\text { Kim } \\
2011 *[12]\end{array}$ & Euro 6 & 1.60 & 2.00 & 1.62 & 0.23 & 0 & 2.59 & 0.367 & 0 & 2.96 & 2.59 & 0.37 & 0.00 & 2.96 \\
\hline $\begin{array}{l}\text { Johnson } \\
2010[15]\end{array}$ & Euro 6 & 1.20 & 2.00 & 3 & 0.15 & 0 & 3.60 & 0.18 & 0 & 3.78 & 3.60 & 0.18 & 0.00 & 3.78 \\
\hline $\begin{array}{l}\text { Desai } \\
\text { 2010[13] }\end{array}$ & Euro 6 & 2.40 & 3.00 & 1.19 & 0.3 & 0 & 2.86 & 0.72 & 0 & 3.58 & 2.86 & 0.72 & 0.00 & 3.58 \\
\hline $\begin{array}{l}\text { Kallinen } \\
2009 *[11]\end{array}$ & Euro 6 & 1.20 & 2.00 & 3.33 & 0 & 0 & 4.00 & 0 & 0 & 4.00 & 4.00 & 0.00 & 0.00 & 4.00 \\
\hline
\end{tabular}

*: loadings specifically designated to a particular standard, see text

**: no loading comparison is available

The regulation standards for the rest of the data are inferred.

$+C V=$ catalyst volume (in litres)

$\neq \mathrm{ED}=$ engine displacement (in litres)

$\wedge M_{t p}^{x}=$ Mass of $\mathrm{x}$ reported in literature

$\wedge \wedge M_{i s o}^{x}=$ Equivalent iso-performance mass of $\mathrm{x}$, adjusted to account for technological progress. 


\section{SI 6) DOC Weight Change and Learning Rate for Different Standard Transitions}

Table SI.6. DOC Weight Change and Learning Rate for Different Standard Transitions

\begin{tabular}{lcccc}
\hline \multicolumn{1}{c}{ Standard Transition } & $\begin{array}{c}\text { Delta Weight } \\
(\mathrm{g})\end{array}$ & $\begin{array}{c}\text { Learning Rate } \\
\%\end{array}$ & $\begin{array}{c}\text { Delta Weight } \\
(\mathrm{g})^{*}\end{array}$ & $\begin{array}{c}\text { Learning Rate } \\
\%^{*}\end{array}$ \\
\hline Euro3-Euro4 & 0.25 & 3.7 & 0.41 & 7.6 \\
Euro4-Euro5 & 0.21 & 3.2 & 0.47 & 7.7 \\
Euro5-Euro6 & 0.38 & 6.9 & 0.44 & 7.6 \\
Average & 0.28 & 4.6 & 0.44 & 7.6 \\
\hline
\end{tabular}

*: values calculated with papers that explicitly designate a particular standard to a specific loading. 
SI 7) Annual Assumed Vehicle Sales by Type (values in millions)

Table SI. 7. Projection of vehicles by drive train used in this work. The projection of future fleet size and make up used in this work comes from the Sustainable Mobility Project (SMP) model developed by the IEA and the World Business Council for Sustainable Development (WBCSD). [See reference 16 for more details about the SMP model.]

\begin{tabular}{cccccc}
\hline Year & Gasoline & $\begin{array}{c}\text { Gas } \\
\text { Hybrid }\end{array}$ & Diesel & $\begin{array}{c}\text { Hybrid } \\
\text { Fuel Cell }\end{array}$ & CNG/LPG \\
\hline $\mathbf{2 0 1 0}$ & 48.4 & 0.22 & 11.3 & 0.006 & 0.96 \\
$\mathbf{2 0 1 1}$ & 49.2 & 0.25 & 11.5 & 0.006 & 0.96 \\
$\mathbf{2 0 1 2}$ & 50 & 0.27 & 11.6 & 0.006 & 0.97 \\
$\mathbf{2 0 1 3}$ & 50.8 & 0.3 & 11.7 & 0.006 & 0.97 \\
$\mathbf{2 0 1 4}$ & 51.6 & 0.33 & 11.9 & 0.006 & 0.98 \\
$\mathbf{2 0 1 5}$ & 52.4 & 0.36 & 12 & 0.007 & 0.98 \\
$\mathbf{2 0 1 6}$ & 53.3 & 0.39 & 12.2 & 0.007 & 0.99 \\
$\mathbf{2 0 1 7}$ & 54.3 & 0.42 & 12.3 & 0.007 & 0.99 \\
$\mathbf{2 0 1 8}$ & 55.2 & 0.45 & 12.5 & 0.007 & 1 \\
$\mathbf{2 0 1 9}$ & 56.2 & 0.49 & 12.7 & 0.007 & 1 \\
$\mathbf{2 0 2 0}$ & 57.1 & 0.52 & 12.9 & 0.007 & 1.01 \\
$\mathbf{2 0 2 1}$ & 58.3 & 0.55 & 13.1 & 0.007 & 1.02 \\
$\mathbf{2 0 2 2}$ & 59.4 & 0.58 & 13.3 & 0.007 & 1.03 \\
$\mathbf{2 0 2 3}$ & 60.6 & 0.61 & 13.5 & 0.008 & 1.03 \\
$\mathbf{2 0 2 4}$ & 61.7 & 0.64 & 13.7 & 0.008 & 1.04 \\
$\mathbf{2 0 2 5}$ & 62.9 & 0.67 & 14 & 0.008 & 1.05 \\
$\mathbf{2 0 2 6}$ & 64.3 & 0.7 & 14.2 & 0.008 & 1.06 \\
\hline $\mathbf{2 0 2 7}$ & 65.7 & 0.74 & 14.5 & 0.008 & 1.07 \\
$\mathbf{2 0 2 8}$ & 67.2 & 0.78 & 14.7 & 0.008 & 1.08 \\
$\mathbf{2 0 2 9}$ & 68.6 & 0.82 & 15 & 0.009 & 1.09 \\
$\mathbf{2 0 3 0}$ & 70.1 & 0.85 & 15.2 & 0.009 & 1.09 \\
\hline
\end{tabular}


SI 8) Annual Assumed Vehicle Sales by Region (values in millions)

The projection of future fleet used in this work comes from the Sustainable Mobility Project (SMP) model developed by the IEA and the World Business Council for Sustainable Development (WBCSD). [See reference 16 for more details about the SMP model.]

\begin{tabular}{cccccccc}
\hline Year & NA & $\begin{array}{c}\text { OECD } \\
\text { Europe }\end{array}$ & $\begin{array}{c}\text { Eastern } \\
\text { Europe }\end{array}$ & China & India & $\begin{array}{c}\text { S Asia } \\
\text { \& } \\
\text { Japan }\end{array}$ & MLA* \\
\hline $\mathbf{2 0 1 0}$ & 17.6 & 17.7 & 4.2 & 3.4 & 1.6 & 9.7 & 6.7 \\
\hline $\mathbf{2 0 1 1}$ & 17.8 & 17.7 & 4.3 & 3.6 & 1.7 & 9.8 & 6.9 \\
$\mathbf{2 0 1 2}$ & 18 & 17.7 & 4.5 & 3.8 & 1.8 & 9.9 & 7.1 \\
\hline $\mathbf{2 0 1 3}$ & 18.1 & 17.7 & 4.6 & 4.1 & 1.9 & 10 & 7.3 \\
$\mathbf{2 0 1 4}$ & 18.3 & 17.7 & 4.8 & 4.3 & 2 & 10.1 & 7.6 \\
\hline $\mathbf{2 0 1 5}$ & 18.4 & 17.8 & 4.9 & 4.5 & 2.1 & 10.2 & 7.8 \\
$\mathbf{2 0 1 6}$ & 18.6 & 17.8 & 5 & 4.8 & 2.2 & 10.4 & 8 \\
\hline $\mathbf{2 0 1 7}$ & 18.8 & 17.8 & 5.2 & 5.1 & 2.3 & 10.5 & 8.3 \\
$\mathbf{2 0 1 8}$ & 19 & 17.8 & 5.4 & 5.4 & 2.5 & 10.6 & 8.5 \\
\hline $\mathbf{2 0 1 9}$ & 19.1 & 17.8 & 5.5 & 5.7 & 2.6 & 10.8 & 8.8 \\
$\mathbf{2 0 2 0}$ & 19.3 & 17.9 & 5.7 & 6.1 & 2.7 & 10.9 & 9 \\
\hline $\mathbf{2 0 2 1}$ & 19.4 & 17.9 & 5.8 & 6.5 & 2.9 & 11.1 & 9.4 \\
$\mathbf{2 0 2 2}$ & 19.6 & 17.9 & 6 & 6.9 & 3 & 11.3 & 9.7 \\
\hline $\mathbf{2 0 2 3}$ & 19.8 & 17.9 & 6.1 & 7.3 & 3.2 & 11.5 & 10 \\
$\mathbf{2 0 2 4}$ & 19.9 & 17.9 & 6.3 & 7.7 & 3.3 & 11.8 & 10.3 \\
\hline $\mathbf{2 0 2 5}$ & 20.1 & 17.9 & 6.4 & 8.1 & 3.5 & 12 & 10.6 \\
\hline $\mathbf{2 0 2 6}$ & 20.2 & 17.9 & 6.6 & 8.6 & 3.7 & 12.3 & 11 \\
\hline $\mathbf{2 0 2 7}$ & 20.4 & 17.9 & 6.8 & 9.2 & 3.9 & 12.5 & 11.4 \\
\hline $\mathbf{2 0 2 8}$ & 20.6 & 17.9 & 6.9 & 9.7 & 4.1 & 12.8 & 11.8 \\
\hline $\mathbf{2 0 2 9}$ & 20.7 & 17.9 & 7.1 & 10.3 & 4.3 & 13.1 & 12.2 \\
$\mathbf{2 0 3 0}$ & 20.9 & 17.9 & 7.3 & 10.8 & 4.5 & 13.4 & 12.5 \\
\hline & & & & & & & \\
\hline & & & & & & \\
\hline
\end{tabular}

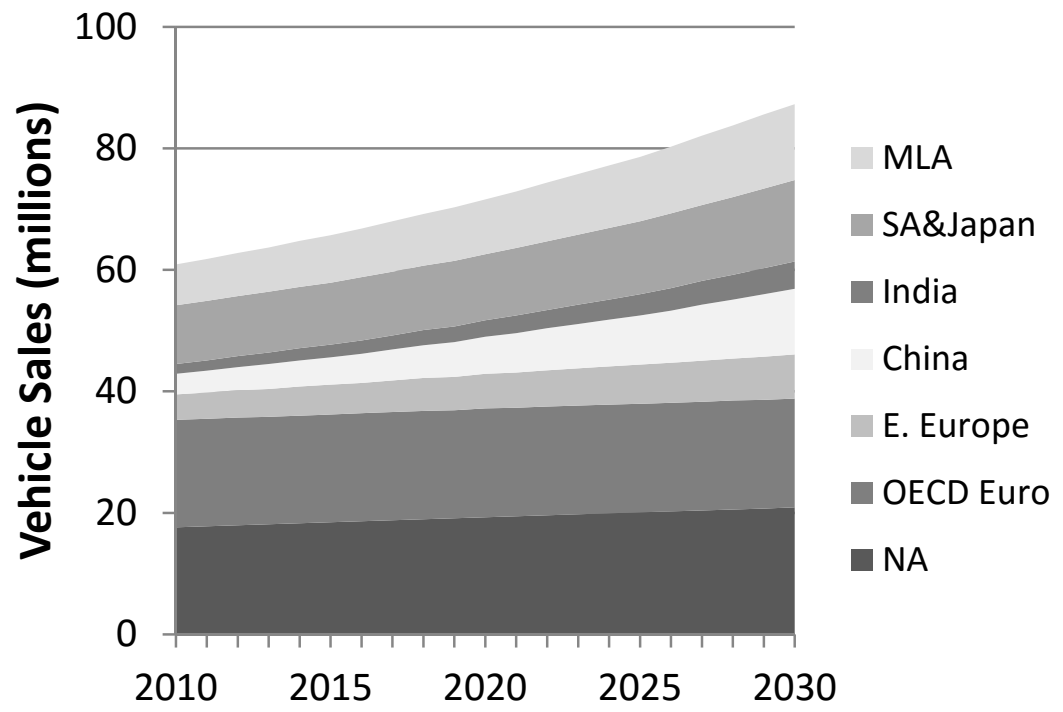

Table SI. 8. Annual Assumed Vehicle Sales by Region (values in millions).

*MLA=Middle East, Latin America, and Africa 


\section{SI 9) References in Supporting Information}

1. Sanchez, F.P., A. Bandivadekar, and J. German, Estimated Cost of Emission Reduction Technologies for Light-Duty Vehicles. 2012, The International Council on Clean Transportation.

2. Zammit, M., et al., The Effects of Catalytic Converter Location and Palladium Loading on Tailpipe Emissions, in SAE 2012 World Congress \& Exhibition. 2012, SAE International: SAE Technical Paper 2012-01-1247.

3. Theis, J.R. and R.W. McCabe, The effects of high temperature lean exposure on the subsequent HC conversion of automotive catalysts. Catalysis Today, 2012. 184(1): p. 262-270.

4. Chatterjee, D., O. Deutschmann, and J. Warnatz, Detailed surface reaction mechanism in a three-way catalyst. Faraday Discussions, 2001. 119(1): p. 371-384.

5. Collins, N.R., et al., Development of Advanced Three-Way Catalysts that Enable Low PGM Loadings for Future Mercosur Emissions Legislation, in SAE Brasil 2002 Congress and Exhibition. 2002, SAE International: SAE Technical Paper 2002-01-3551.

6. Mitchell, G., D. Ball, and M. Zammit. PGM Optimization by Robust Design. in SAE Powertrain \& Fluid Systems Conference and Exhibition. 2005. San Antonio, TX.

7. Matsuzono, Y., et al., Development of Advanced and Low PGM TWC System for LEV2 PZ EV and LEV3 SULEV30, in SAE 2012 World Congress \& Exhibition. 2012, SAE International: SAE Technical Paper 2012-01-1242.

8. Hangas, J. and A.E. Chen, Comparative Analytical Study of Two Pt-Rh Three-way Catalysts. Catalysis Letters, $2006.108(1-2)$ : p. 103-111.

9. Glover, L., et al., Performance Characterization of a Range of Diesel Oxidation Catalysts: Effect of Pt:Pd Ratio on Light Off Behaviour and Nitrogen Species Formation, in 10th International Conference on Engines \& Vehicles. 2011: SAE Technical Paper 2011-24-0193.

10. Han, M., D. Assanis, and S. Bohac, Characterization of Heat-up Diesel Oxidation Catalysts through Gas Flow Reactor and in-situ Engine Testing. Journal of Automobile Engineering, 2007. 2008: p. 1705-1715.

11. Kallinen, K., et al., Pt/Pd Diesel Oxidation Catalyst : A Study on the Properties Enhanced by the Use of Pd, in SIAT 2009. 2009, The Automotive Research Association of India: SAE Technical Paper: 2009-26-0018.

12. Kim, C.H., et al., The Effect of Pt-Pd Ratio on Oxidation Catalysts Under Simulated Diesel Exhaust, in SAE 2011 World Congress \& Exhibition. 2011, SAE International: SAE Technical Paper 2011-01-1134.

13. Desai, M.R., et al., Modeling of Non-Road Diesel Exhaust Aftertreatment Systems: Diesel Oxidation and Selective Catalytic Reduction Catalysts, in SAE 2010 Powertrains Fuels \& Lubricants Meeting. 2010: SAE Technical Paper 2010-01-2092.

14. Wiebenga, M.H., et al., Deactivation mechanisms of Pt/Pd-based diesel oxidation catalysts. Catalysis today, $2012.184(1)$ : p. 197-204.

15. Johnson, T.V., Review of Diesel Emissions and Control. SAE Technical Paper, 2010. 3: p. 16-29.

16. Fulton, L. and G. Eads, IEA/SMP Model Documentation and Reference Case Projection. 2004, International Energy Agency (IEA) / World Business Council for Sustainable Development (WBCSD). 